\title{
Calibration of the Normalized Radar Cross Section for Sentinel-1 Wave Mode
}

\author{
Li Huimin ${ }^{1}$, Mouche Alexis ${ }^{2}$, Stopa Justin E. ${ }^{3}$, Chapron Bertrand ${ }^{4}$
}

${ }^{1}$ Univ. Brest, CNRS, IRD, Laboratoire d'Océanographie Physique et Spatiale, L'Institut Français de Recherche pour l'Exploitation de la Mer, IUEM, 29280 Brest, France, and also with IMT Atlantique, LabSTICC, UBL, 29238 Brest, France

${ }^{2}$ Univ. Brest, CNRS, IRD, Laboratoire d'Océanographie Physique et Spatiale, L'Institut Français de Recherche pour l'Exploitation de la Mer, IUEM, 29280 Brest, France.

${ }^{3}$ Department of Ocean Resources and Engineering, University of Hawaii at Manoa, Honolulu, HI 96822 USA.

${ }^{4}$ Univ. Brest, CNRS, IRD, Laboratoire d'Océanographie Physique et Spatiale, L'Institut Français de Recherche pour l'Exploitation de la Mer, IUEM, 29280 Brest, France.

Email addresses : huimin.li@ifremer.fr ; alexis.mouche@ifremer.fr ; justin.stopa@ifremer.fr ; bertrand.chapron@ifremer.fr

\begin{abstract}
:
Sentinel-1 (S-1) is a two-satellite constellation for continuity of operational synthetic aperture radar (SAR) observations. Wave mode (WV) is the default mode over open ocean for S-1 to monitor global ocean waves and wind field. Therefore, proper radiometric calibration is essential to accurately infer these geophysical quantities. Based on the global data set acquired by S-1A WV, assessment of normalized radar cross section (NRCS) is carried out through comparison with CMOD5.N predictions over open ocean. The calibration accuracy quantified by NRCS residuals between SAR measurements and CMOD5.N demonstrates distinct features for two incidence angles $\left(23.8^{\circ}\right.$ and $\left.36.8^{\circ}\right)$. Particularly, NRCS at $23.8^{\circ}$ is overall consistent with CMOD5.N, while NRCS at $36.8^{\circ}$ displays great deviation. Two recalibration methods are then implemented by examining the backscattering profile over Amazon rain forest and ocean calibration. Both methods show the necessity for recalibration and obtain comparable correction factors for WV1 and WV2, respectively. The NRCS residuals by applying both methods are significantly reduced toward zero. By comparison, ocean calibration is more efficient and practical to implement.
\end{abstract}




\section{INTRODUCTION}

$\mathbf{S}$ ENTINEL-1 (S-1) is the new Synthetic Aperture Radar (SAR) constellation launched and operated by the $\mathrm{Eu}-$ ropean Space Agency (ESA), consisting of two identical platforms (S-1A and S-1B). S-1A and S-1B were launched in April 2014 and May 2016 and are operational since October 2015 and July 2016 respectively. There are four imaging modes which operate in C-band: Extra Wide-swath (EW), Interferometric Wide swath (IW), Stripmap (SM) and WaVe mode (WV) [1]. WV is the highest spatial resolution mode, with nominal spacing of $4 \mathrm{~m}$ and a footprint of $20 \times 20 \mathrm{~km}$. The WV was designed to specifically measure ocean waves and ocean surface winds on a global scale [2], [3], [4].

Over ocean the normalized radar cross section (NRCS or $\sigma^{0}$ used inter-changeably through this work) responds primarily to the ocean surface wind vector. Proper calibration of the WV's NRCS is necessary in order to accurately estimate geophysical quantities such as oceanic wind speeds [5], [2] and sea state

Huimin Li, Alexis Mouche, Justin Stopa and Bertrand Chapron are with Univ. Brest, CNRS, IRD, Ifremer, Laboratoire d'Océanographie Physique et Spatiale (LOPS), IUEM, 29280, Brest, France.

This work is supported by Sentinel-1 A Mission Performance Center (4000107360/12/I-LG). Huimin Li would like to thank for financial support of China Scholarship Council (CSC).

E-mail: huimin.li@ifremer.fr; justin.stopa@ifremer.fr; bertrand.chapron@ifremer.fr

Huimin Li is also with IMT Atlantique, Lab-STICC, UBL, 29238 Brest, France. parameters, such as significant wave height [6], [7], [8] or ocean swell spectrum [9]. In general, the radiometric calibration of NRCS is performed by comparing the backscatter from ground targets with known NRCS that are concurrently measured by transponders [10], [11] or routine acquisitions over the reference distributed targets such as Amazon rainforest [12], [13].

Prior to any geophysical applications over the ocean, it is essential to assess accuracy of its radiometric calibration. However, the lack of sufficient acquisitions over ground transponders or over the rain-forest makes the calibration difficult. Therefore we use an alternative method to quantitatively assess NRCS calibration through comparisons with the an empirical Geophysical Model Function (GMF) combined with collocated 10 meter height wind (U10) from the European Centre for Medium-range Weather Forecasts (ECMWF) forecast. In this way, we take advantage of the systematic and regular WV acquisitions over open ocean to calibrate the NRCS. Here, we use CMOD5.N, the state-of-the-art GMF, to estimate the predicted NRCS as a function of wind vector, incidence and antenna look angle.

Beyond the NRCS calibration assessment, this work aims at providing a robust method for users to re-calibrate the NRCS from S-1 WV, which enables more precise measures of geophysical parameters over the ocean. Thus, we propose two different and complementary methods for recalibration. The first method relies on the use of the Amazon rain-forest through the Gamma-nought parameter $\gamma^{0}$ [14], [15], [13] whereas the second is an evaluation over open ocean [16].

The manuscript is organized as follows. We describe the collocated dataset between S-1A WV and ECMWF winds as well as computation of S-1 NRCS in Section 2. In section 3 we assess the current ESA S-1 NRCS relative to the ECMWF winds. Then, in section 4 , we present our strategy to recalibrate the NRCS and we provide calibration factors. Discussions and conclusions follow in sections 5 and 6 respectively.

\section{DATA AND NRCS}

\section{A. Collocated Sentinel-1A and ECMWF wind speed}

S-1 WV alternates between WV1 $\left(23.8^{\circ}\right)$ and WV2 $\left(36.8^{\circ}\right)$ with a new 'leap frog' approach [17]. Each vignette is acquired every $100 \mathrm{~km}$ along the flight direction [4]. S-1 WV can only be operated in single polarization (either VV or $\mathrm{HH}$ ) for a given acquisition. The $\mathrm{WV}$ at $\mathrm{VV}$ polarization is the default mode over global ocean. Here we use 27,000 images acquired between June 2016 and June 2017 by S-1A WV1 and WV2 at VV polarization, respectively. This period was 

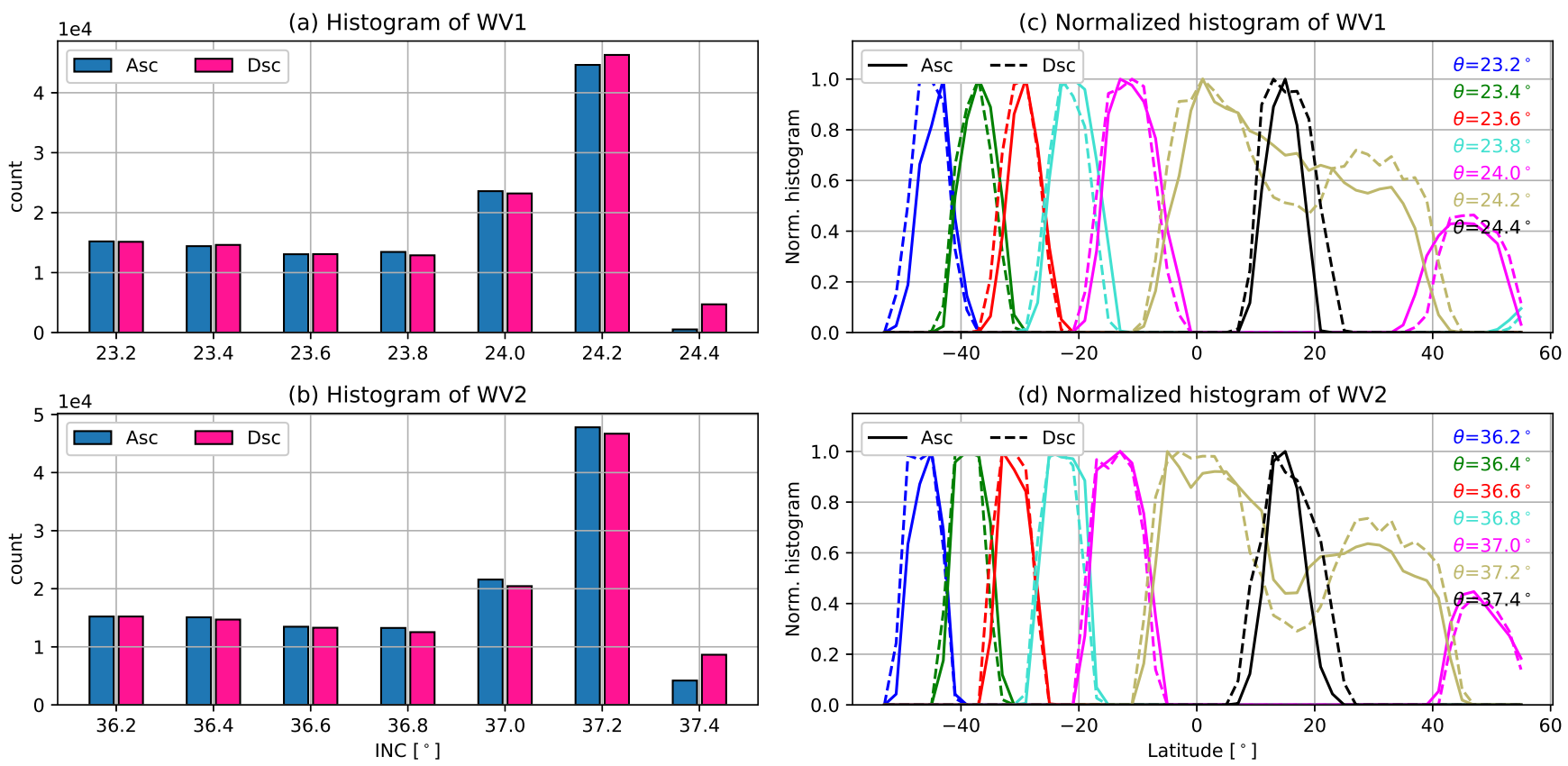

Fig. 1. Histogram of incidence angles for (a) WV1 and (b) WV2, receptively. Bin size of incidence angle is $0.22^{\circ}$. (c) Normalized histogram of latitude at given incidence angle for WV1. Bin size of latitude is $2^{\circ}$. Solid lines are for Ascending pass and dashed lines are for Descending pass. Colors represent different incidence angles. Products acquired at latitude higher than $55^{\circ}$ have been filtered out to avoid ice contamination.

chosen to avoid processor updates, such as one that occurred in May 2016, and to maintain the consistency of instrument calibration. We also limit the data to latitudes less than $55^{\circ}$ to avoid any possible sea-ice contaminations. Each WV imagette is collocated with ECMWF forecast winds from operational forecast model. The wind dataset is considered 'nowcast' and is a compiled dataset of the ECWMF forecast initialized every 6 hours. The ECWMF winds are available every 3 hours on a spatial grid of $0.25^{\circ}$. The collocation takes the nearest point both in space and in time, resulting in maximum spatial distance of $12.5 \mathrm{~km}$ and maximum temporal difference being $1 \mathrm{~h} 30$.

$\mathrm{A} \pm 0.7^{\circ}$ incidence angle variation is observed for S- $1 \mathrm{WV} 1$ and WV2 along the orbit. Fig.1 (a) and (b) present histogram of collocated dataset binned by incidence angle for WV1 and WV2, respectively with bin size of $0.2^{\circ}$. For each incidence bin, the numbers of products are regular (around 15,000) at incidence smaller than $24.0^{\circ}\left(37.0^{\circ}\right)$ for WV1 (WV2). Note that $24.2^{\circ}\left(37.2^{\circ}\right)$ for WV1 (WV2) has the maximum of acquisitions around 41,000. Only about 5,000 imagettes are acquired at incidence of $24.4^{\circ}\left(37.4^{\circ}\right)$ for WV1 (WV2). Ascending and descending passes have similar data counts (except $24.4^{\circ}\left(37.4^{\circ}\right)$ for WV1 (WV2)).

Incidence angles are not evenly distributed across the globe. This is shown by the normalized histograms in Fig.1 (c) and (d), where latitude bin size is $2^{\circ}$. There is an incidence angle dependence on latitude for both ascending and descending passes. Both ascending and descending passes have similar spread over latitude per incidence. Incidence angle generally increases from the south to the north. In particular, the highest incidence angles plotted as black lines $\left(24.4^{\circ}\right.$ for WV1 and $37.4^{\circ}$ for WV2) are distributed between the Equator $\left(0^{\circ}\right)$ and $20^{\circ} \mathrm{N}$. Incidence angles of $24.2^{\circ}$ for WV1 and $37.2^{\circ}$ for WV2 have the largest spread over $10^{\circ} \mathrm{S}$ to $40^{\circ} \mathrm{N}$. Since wind speed and direction vary greatly with latitude [18], WV1 and WV2 data are analyzed separately throughout this work. Ascending and descending passes are merged and analyzed as a single dataset since we did not find any differences related to the orbit configuration.

\section{B. Normalized radar cross section}

Over ocean, at C-band the NRCS is a function of radar polarization, incidence angle as well as environmental conditions such as the wind field [19]. For a given polarization and wind speed, the NRCS decreases with increasing incidence angle. As such, the signal-to-noise ratio decreases with incidence angles, leading to possible contamination of thermal noise at high incidence angle and under low wind conditions. A proper noise-correction is therefore essential to obtain an accurate NRCS. The radiometric calibration of S-1A taking noisecorrection into account is expressed as [20] :

$$
\sigma^{0}=\frac{D N^{2}-\eta_{i}}{A_{i}^{2}}
$$

where $D N=\sqrt{\left(I^{2}+Q^{2}\right)}$ is the digital number of ESA Level-1 Single Look Complex (SLC) product. $\eta$ is the de-noise Look-Up-Table (LUT) provided in annotation file and $A_{i}$ is the calibration LUT for $\sigma^{0}$ as function of azimuth and range pixel, also annotated in the Level-1 products. Multiple calibration procedures are incorporated in $A_{i}$, containing area normalization factor, calibration constant and geophysical calibration. Among which, the geophysical calibration constant, the NRCS difference between SAR measurements and simulated NRCS 
using CMOD-IFR2 from collocated ECMWF winds in the range of $[4 \mathrm{~m} / \mathrm{s}, 10 \mathrm{~m} / \mathrm{s}]$, is provided by the Sentinel-1 Mission Performance Center for WV1 and WV2, respectively [21].

In this study, we compute a single $\sigma^{0}$ per imagette at a resolution of $20 \mathrm{~km}$ by $20 \mathrm{~km}$. The noise equivalent sigmanought (NESZ) is further removed from NRCS in Eq. (1). The NESZ is defined as the maximum value of histogram for NRCS with collocated wind speed lower than $1 \mathrm{~ms}^{-1}$. We obtain 0.0001 for WV1 and 0.00156 for WV2 in linear unit. The NESZ-corrected NRCS is used throughout rest of this manuscript unless particularly stated. Images with negative NRCS are not included in the analysis because the backscattered signal is lower than than thermal noise.

\section{CMOD5.N}

The empirical GMF, CMOD5.N, was developed for global applications for use of the C-band scatterometer on board ERS-2 and Advanced Scatterometer (ASCAT) [22]. CMOD5.N performs better than its predecessor CMOD5 with better wind retrieval accuracy [23], [24]. It is routinely used for operational ocean surface wind produced by the OSI-SAF (Ocean and Sea Ice - Satellite Application Facilities) [25]. CMOD5.N is valid for incidence angles ranging from $18^{\circ}$ to $60^{\circ}$, covering the two incidence angles of WV. CMOD5.N relates the NRCS to the incidence angle, wind speed and wind direction (relative to radar line-of-sight), and polarization under neutral atmospheric stability with the following equation [22]:

$$
\sigma^{0}=B_{0}\left[1+B_{1} \cos (\phi)+B_{2} \cos (2 \phi)\right]
$$

where $\phi$ is the wind direction relative to the antenna look angle. $B_{0}$ is the dominant term determining scale of NRCS for given wind speed. $B_{1}$ incorporates the up-downwind asymmetry of NRCS, while $B_{2}$ expresses the up-crosswind asymmetry of NRCS. The three terms are all functions of incidence angle, wind speed, and wind direction. Coefficients for each term are given in [22]. It is used throughout the rest of the manuscript to predict NRCS for given S-1A incidence angle and collocated ECMWF winds on a case-to-case basis.

\section{ASSESSMENT OF THE NORMALIZED RADAR CROSS SECTION}

S-1A WV NRCS is assessed for given wind speed and incidence angle by comparing with the predicted value from CMOD5.N. The deviations are then quantified by NRCS residual between SAR measurements and CMOD5.N prediction.

First we evaluate the S-1A NRCS relative to wind direction for given wind speed and incidence angle. U10 of $12 \mathrm{~ms}^{-1}$ is used to illustrate the comparison of S-1A NRCS with the CMOD5.N prediction. We choose $\mathrm{U} 10=12 \mathrm{~ms}^{-1}$ because it is a compromise between sufficiently high signal-to-noise ratio and adequate collocation pairs. In addition, working with incidence angles of $23.4^{\circ}$ and $36.4^{\circ}$ ensures a narrow spread over latitudes as well as relatively uniform wind direction across $\left[0^{\circ}, 360^{\circ}\right]$. NRCS with respect to wind direction is shown on Fig. 2 (a) for WV1 and on Fig. 2 (b) for WV2. Within each direction bin of $10^{\circ}, 50$ data points are randomly
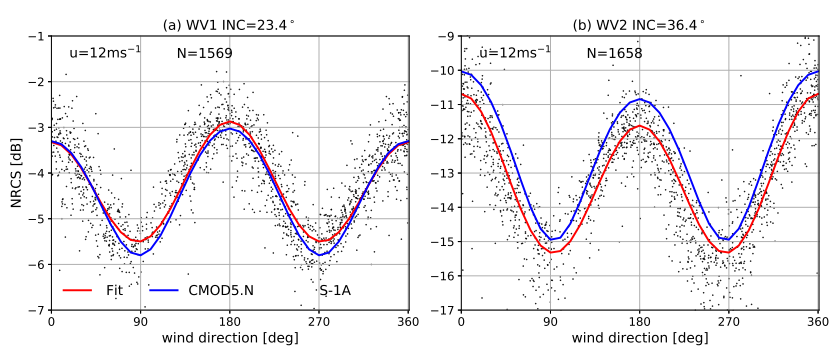

Fig. 2. NRCS at $12 \mathrm{~ms}^{-1}$ relative to wind direction for (a) WV1 at incidence of $23.4^{\circ}$; (b) WV2 at incidence of $36.4^{\circ}$. Black dots are S-1A measurements and red lines are fit to S-1A observed NRCS in form of Eq.(2). Blue line is the predicted CMOD5.N for given incidence angle and wind speed. For wind direction, $0^{\circ}$ indicates upwind and $180^{\circ}$ is downwind.

selected if there are more than 50 samples. Otherwise, all data points are used for that bin. This equalization method is used to remove biases induced by nonuniform distribution of wind direction [26]. This results in 1569 and 1658 points (marked by 'N') for WV1 and WV2, respectively for $\mathrm{U} 10=12 \mathrm{~ms}^{-1}$. The random selection taken in the equalization procedure has been repeated for several times and we found the nearly identical results.

As shown in Fig.2 (a), the fit to S-1A measurements are overall in good agreement with CMOD5.N curve for WV1. In particular, S-1A NRCS are slightly greater than CMOD5.N estimate approximately by about $0.3 \mathrm{~dB}$ at crosswind (wind direction of $90^{\circ}$ ). Similar trend is found for other incidence angles of WV1. By contrast, as shown in Fig.2 (b), S-1A NRCS is constantly smaller than the CMOD5.N estimate across all wind directions for WV2. The NRCS residual $\left(\sigma_{S A R^{-}}^{0}\right.$ $\left.\sigma_{C M O D 5 . N}^{0}\right)$ at crosswind is $-0.4041 \mathrm{~dB}$ and $-0.6545 \mathrm{~dB}$ at upwind. Similar trend has been found for wind speeds higher than $7 \mathrm{~ms}^{-1}$ for other incidence angles of WV2. This suggests that the WV has not been properly calibrated for WV2 and wind speeds would be consistently underestimated.

CMOD5.N is now systematically used to calculate the expected NRCS for each S-1A imagette based on collocated ECMWF winds and given incidence angle. The NRCS residuals $\left(\sigma_{S A R}^{0}-\sigma_{C M O D 5 . N}^{0}\right)$ relative to U10 at three incidence angles for WV1 (WV2) are presented in the upper (lower) panels of Fig.3. The main similarities between WV1 and WV2 are the slightly decreasing NRCS residual with increasing wind speed up to $7 \mathrm{~ms}^{-1}$ before remaining steady at higher winds. The NRCS residuals for WV1 and WV2 are different when $\mathrm{U} 10>7 \mathrm{~ms}^{-1}$. Overall the average NRCS residual is approximately $0 \mathrm{~dB}$ for all $\mathrm{WV} 1$ and $-0.8 \mathrm{~dB}$ for WV2 when considering all incidence angles. In particular, for $\mathrm{U} 10=12 \mathrm{~ms}^{-1}$, there are NRCS residuals of $0.05 \mathrm{~dB}$ for WV1 (incidence angle of $23.6^{\circ}$ ) and $-0.88 \mathrm{~dB}$ for WV2 (incidence of $36.6^{\circ}$ ) (see Fig. 3(b),(e)). In addition, the standard deviation exhibits similar variation trend with the mean residual. The greater variability at low wind speed $\left(<3 \mathrm{~ms}^{-1}\right)$ is mostly due to limited number of observations.

The negative NRCS residuals are unexpected for WV2. There are several possible sources of error to verify, including accuracy of radiometric calibration for WV2, bias of NESZ correction as well as errors in collocated ECMWF winds. The 
(a) WV1 $23.2^{\circ}+0.1^{\circ}$

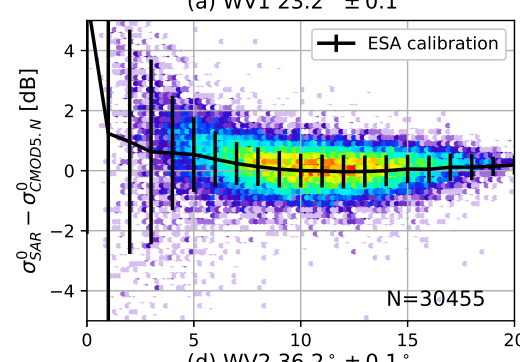

(d) WV2 $36.2^{\circ} \pm 0.1^{\circ}$



(b) WV1 $23.6^{\circ} \pm 0.1^{\circ}$

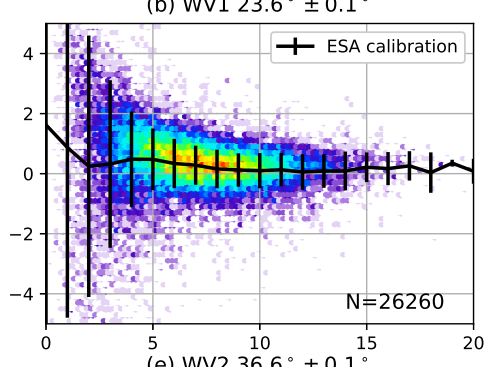

(e) WV2 $36.6^{\circ} \pm 0.1^{\circ}$

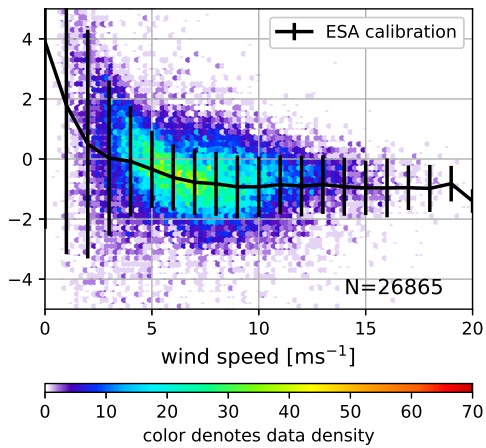

(c) WV1 $24.2^{\circ}+0.1^{\circ}$

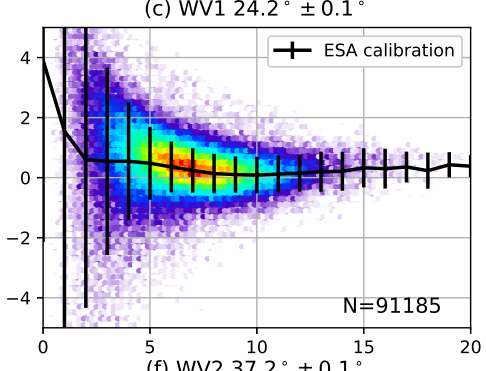

(f) $W V 237.2^{\circ} \pm 0.1^{\circ}$

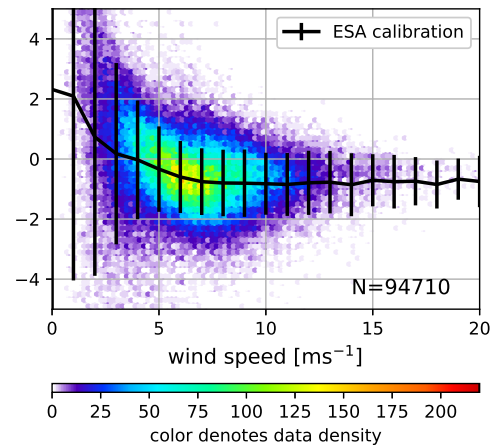

Fig. 3. NRCS difference ( $\left.\sigma_{S A R^{-}}^{0} \sigma_{C M O D 5 . N}^{0}\right)$ as function of wind speed for given incidence angle for (top) WV1; (bottom) WV2. Solid line is mean NRCS difference with bin size of $1 \mathrm{~ms}^{-1}$. The error bar represents 1 standard deviation of NRCS difference within each bin. Colors denote count of data points.

bias of NESZ could be ruled out because higher wind speed corresponds to higher signal-to-noise ratio and consequently leads to negligible noise contribution. Indeed the contribution of thermal noise to NRCS is expected to decrease with increasing wind speed, making it impossible to reproduce the increasing deviation. As for biases induced by the ECMWF winds, WV1 and WV2 used the same data source, so that any errors in the ECMWF winds should be equally translated into NRCS residuals for both WV1 and WV2. However, we do not see this behavior. Therefore the calibration is likely the best candidate for causing the NRCS discrepancies. In the following, we apply two different methods to re-calibrate S-1A WV NRCS and compare their respective performances.

\section{RE-CALIBRATION OF WV AND VALIDATION}

In this section, we first examine the $\gamma^{0}$ profile over rainforest and then the NRCS residuals over open ocean.

\section{A. Re-calibration over rain-forest}

For radiometric calibration of most scatterometers and SARs [14], [27], [28], the Amazon rain-forest is used as a reference distributed target to monitor variation of backscattering during missions lifetime. For C-band radar, this target could be considered as a rough surface, which equally scatters the incident radar electromagnetic waves in all directions. Therefore the backscatter has small incidence angle dependence and can be characterized by [28]:

$$
\gamma^{0}=\sigma^{0} / \cos (\theta)=\beta^{0} \tan (\theta)
$$

where $\gamma^{0}, \sigma^{0}$ and $\beta^{0}$ are different forms to express the backscatter signal. In terms of the isotropic properties of rain-forest, $\gamma^{0}$ can be approximated incidence angle independent [14]. This property together with the stability of rain-forest allow us to directly compare measurements from different imaging modes of S-1A that have large range of incidence angles.

IW $\gamma^{0}$ is chosen as reference to re-calibrate WV because IW is well calibrated against ground corner reflectors [29]. Besides, the comparison of IW NRCS with CMOD5.N using collocated ECMWF winds for various incidence angles shows good consistency with mean NRCS residual around $0.1 \mathrm{~dB}$ (not shown). Adopting the common test site used by scatterometer community [15], we collect IW acquisitions from $4^{\circ} \mathrm{S}$ to $9^{\circ} \mathrm{S}$ in latitude and from $73^{\circ} \mathrm{W}$ to $59^{\circ} \mathrm{W}$ in longitude between June 2016 and June 2017. In total, 425 IW Ground Range Detected (GRD) products are used in this study, each with spatial coverage of $250 \mathrm{~km}$ by $250 \mathrm{~km}$. In contrast, there are fewer acquisitions by WV over this test site. Most of the WV images are located in the eastern South America of relatively high heterogeneity, which would induce spatial variability in radar backscatter. To assure the spatial homogeneity of imaged area, we manually screened 366 products acquired by WV1 and 338 by WV2 over rain-forest. Both IW and WV products are processed into sub-images of $10 \mathrm{~km}$ by $10 \mathrm{~km}$. An example of WV2 imagette is shown in Fig. 4 (a). For each subimage, histogram of $\gamma^{0}$ is computed and fitted with a normal distribution plus a second-order polynomial, expressed as [15]:

$$
F(x)=A_{0} \cdot \exp \left[-\frac{\left(x-A_{1}\right)^{2}}{2 A_{2}^{2}}\right]+A_{3} \cdot x^{2}+A_{4} \cdot x+A_{5}
$$

where $x$ denote $\gamma^{0}$ and $A_{i}$ are six coefficients to be determined, which are determined by non-linear least squares method. Fig. 4 (b) is an example of $\gamma^{0}$ histogram over the sub-image I in Fig. 4 (a). The coefficient $A_{1}$ is then taken as 



Fig. 4. (a) An example of processing one imagette into subimages of $10 \mathrm{~km}$ by $10 \mathrm{~km}$ (WV2 image acquired on November 10, 2017 at 22:21:41 UTC). (b) Histogram of $\gamma^{0}$ over sub-image I (blue solid line) and fit (red dashed line) with Eq.(4). The bin size of $\gamma^{0}$ is $0.1 \mathrm{~dB}$. The vertical dashed line represents position of fitted $A_{1}$ coefficient.

the reference $\gamma^{0}$ over this sub-image. The same procedure is applied to all WV and IW products.

The geographical positions of processed IW and WV data are presented in Fig. 5 (a). As shown, IW data are densely located in the west, while points of WV are sparsely distributed throughout. The weekly count of data points is shown in Fig. 5 (b). Although there is one to two orders of magnitude difference in the number of data counts between WV and IW, both show regular acquisition number during the study period, without obvious seasonal variation. This could to an extent avoid a temporal bias caused by nonuniform acquisitions.

As theoretically derived, $\gamma^{0}$ over Amazon rain-forest is approximately a constant for VV polarization, independent of elevation angle or incidence angle[28]. Though, it is preferable to compare the $\gamma^{0}$ observed at the same elevation angle in purpose to maintain the same antenna elevation pattern. Elevation angle of IW varies between $27.5^{\circ}$ and $40.5^{\circ}$, covering WV2 $\left(32.6^{\circ}\right)$ but does not cover WV1 $\left(21.6^{\circ}\right)$. For WV2, a filtering of elevation angle within $32.6^{\circ} \pm 0.4^{\circ}$ is accordingly applied to processed IW data points. While for WV1, we use a different strategy. IW $\gamma^{0}$ relative to elevation angle is shown in Fig.6 (a). It is worth noting that the $\gamma^{0}$ does exhibit variation with elevation angle, which might be caused by the inadequate correction of inter-beam as well as beam-to-beam gain offset of azimuth antenna pattern as reported in [29]. In this study, we choose to use all IW Level-1 data given the small variability of $\gamma^{0}$, assuming that the $\gamma^{0}$ dependence on the elevation angle is negligible.
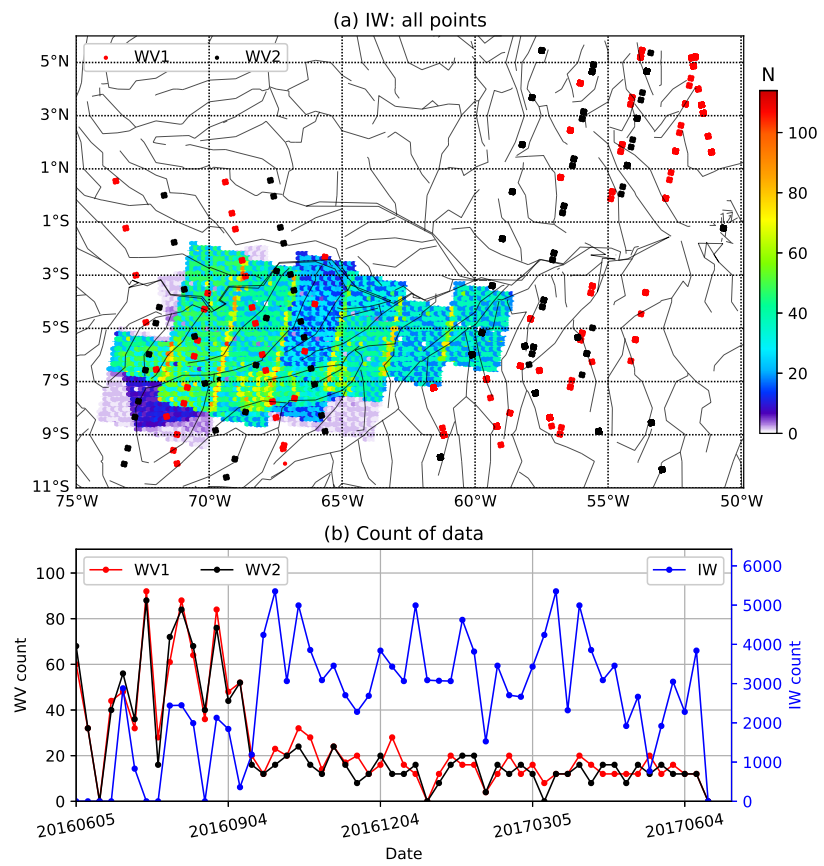

Fig. 5. (a) Map of processed IW and WV points. Colors denote number of processed IW points. The solid circle represents position of WV data points (WV1 in red and WV2 in black). (b) Weekly count of data points for WV and IW, respectively.

We present the histograms of $\gamma^{0}$ for all IW points, filtered with respect to elevation angle, WV1, and WV2, in Fig. 6 (be). The formula in Eq. (4) is employed to fit the histogram and shown as black dashed lines. The fitted coefficient $A_{1}$ is also annotated in the plots. By comparing $A_{1}$ in Fig. 6(b) and (d), there is a difference of $0.2803 \mathrm{~dB}$ between IW and WV1, while $0.4449 \mathrm{~dB}$ between filtered IW and WV2 (Fig. 6c,e). A seasonal variation on the order of $0.15 \mathrm{~dB}$ [14] has been commonly observed by scatterometers. However, this cannot explain the difference found here. For simplicity, the seasonal variation is not considered for the moment in this re-calibration process. In order to make the $\gamma^{0}$ consistent between IW and WV1, a deduction of $0.2803 \mathrm{~dB}$ is required which is equivalent to be divided by a factor of 1.0667 in linear units. Similarly, for WV2 $\gamma^{0}$ a deduction of $-0.4449 \mathrm{~dB}$ is equivalent to be divided by 0.9026 in linear units.

\section{B. Ocean calibration}

Although the $\gamma^{0}$ profile over Amazon rain-forest could serve to re-calibrate WV NRCS, few WV products limits its routine application. A more practical method taking advantage of the numerous acquisitions over open ocean is therefore advantageous. The ocean calibration procedure [16] is widely used in scatterometer community to derive the NRCS corrections. It is more feasible since it only needs a few days of collocated pairs to compute the correction constant.

The ocean calibration algorithm is applied to the collocated dataset between S-1A and ECMWF winds. The dataset is split into wind speed bins of $1 \mathrm{~ms}^{-1}$ and wind direction bin of $10^{\circ}$. Within each wind speed bin, one out of thirty-six of the collocated pairs is randomly selected to equalize wind 

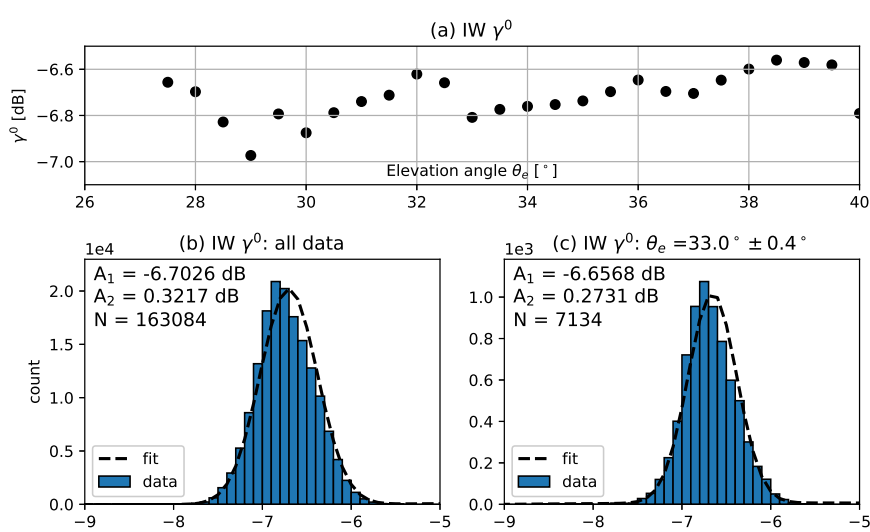

le3 (c) IW $\gamma^{0}: \theta_{e}=33.0^{\circ} \pm 0.4^{\circ}$


Fig. 6. (a) Variation of $\gamma^{0}$ relative to elevation angle. Histogram of $\gamma^{0}$ for (b) all IW data; (c) IW data filtered around WV2 elevation angle; (d) WV1; (e) WV2. The fit formula to histogram is given in Eq.(4), plotted by black dashed lines. The fitted $A_{1}$ coefficients are annotated in subplots.

direction bin. This varying threshold considerably preserves the proportion of dataset relative to wind speed. An averaged NRCS residual is calculated per wind speed, which is further weighted by the occurrence of this wind speed to compute the final NRCS residual. It is worth noting here that only the cases with collocated ECMWF wind speed higher than $1 \mathrm{~ms}^{-1}$ are used to estimate the final NRCS residual. Based on the collocated dataset, the final NRCS residuals are $0.2730 \mathrm{~dB}$ for WV1 and $-0.5750 \mathrm{~dB}$ for WV2, which correspond to a correction constant of 1.0649 for WV1 and 0.8760 for WV2 to divide in linear unit. These ocean correction constants are comparable with those obtained using Amazon rain-forest calibration method. This confirms the robustness of the ocean calibration procedure for SAR WV data.

\section{Assessment of re-calibrated NRCS}

It should be noted that both re-calibration methods are not devoted to seeking an absolute radiometric calibration constant for WV. Its purpose is to tune an additional correction factor based on ESA provided NRCS. As expressed in Eq. (3), the two backscattering coefficients $\gamma^{0}$ and $\sigma^{0}$ in linear unit share the same radiometric calibration constant. As such, the additional factors are supposed to be further divided by ESAcalibrated $\sigma^{0}$ to obtain the re-calibrated NRCS.

Fig. 7 presents the re-calibrated NRCS curve relative to wind direction at wind speed of $12 \mathrm{~ms}^{-1}$ as shown in Fig. 2. Both rain-forest calibration (black lines) and ocean calibration (red lines) are plotted. It is found that the agreement between re-calibrated NRCS and CMOD5.N based estimates improves in contrast to Fig. 2. Particularly for WV2, the NRCS residual
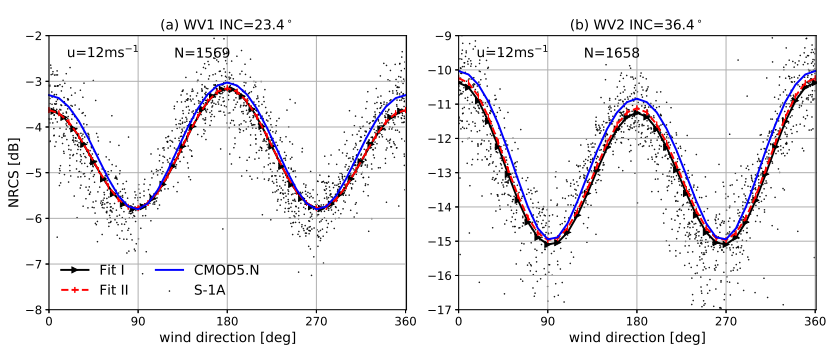

Fig. 7. The same as Fig.2 but for re-calibrated NRCS. The scatters are re-calibrated NRCS using rain-forest method. Black line (marker of right triangle) 'Fit I' represents rain-forest calibration method and red line (marker of vertical line) 'Fit II' for ocean calibration.

is reduced to about $-0.24083 \mathrm{~dB}$ for rain-forest method and $-0.1109 \mathrm{~dB}$ for ocean calibration at crosswind (wind direction of $90^{\circ}$ ). This improvement is further quantified by the recalibrated NRCS residuals shown in Fig. 8. The blacks lines are identical to Fig. 3 for reference. For WV1, the fit for rain-forest calibration (blue line with right triangle) are superimposed by the fit lines of ocean calibration (red with error bar). The error bar represents standard deviation of NRCS difference within each bin. The outliers when $U 10>15 \mathrm{~ms}^{-1}$ in Fig. 8 (e) are caused by the limited number of data points. By comparison, both re-calibrated NRCS residuals using either rain-forest or ocean calibration demonstrate reduced deviation from zero, particularly at high wind. For WV2 in Fig. 8 (d-f), the mean NRCS residuals show varying trend relative to wind speed with incidence angles. For incidence angles of $36.6^{\circ}$ and $37.2^{\circ}$ in Fig.8(e) and (f), the residual is close to zero with negligible variation on the order of $-0.05 \mathrm{~dB}$. However, clearly decreasing NRCS residuals with increasing wind speed is still found at $36.2^{\circ}$ in Fig.8 (d). The reasons responsible for this variation are discussed in the next section. To summarize the overall performance of both re-calibration methods, the mean NRCS residuals of the whole dataset before and after recalibration are listed in Table I. The two re-calibration methods have comparable performance and improve the mean residual for both WV1 and WV2 in comparison to ESA calibration.

TABLE I

MEAN NRCS RESIDUAL

\begin{tabular}{|c|c|c|}
\hline NRCS Residual & WV1 $[\mathrm{dB}]$ & WV2 $[\mathrm{dB}]$ \\
\hline ESA calibration & 0.2541 & -0.6052 \\
\hline rain-forest calibration & -0.0241 & -0.1642 \\
\hline ocean calibration & -0.0169 & -0.0352 \\
\hline
\end{tabular}

\section{DISCUSSION}

Despite the small amount of WV data acquired over the Amazon rain forest, the conventional analysis of $\gamma^{0}$ over this area has shown that a correction factor is essential to re-calibrate radar backscatter for both WV1 and WV2. In this study, we also showed that the significant number of collocated data between S-1A WV acquired over open ocean 
(a) WV1 $23.2^{\circ} \pm 0.1^{\circ}$

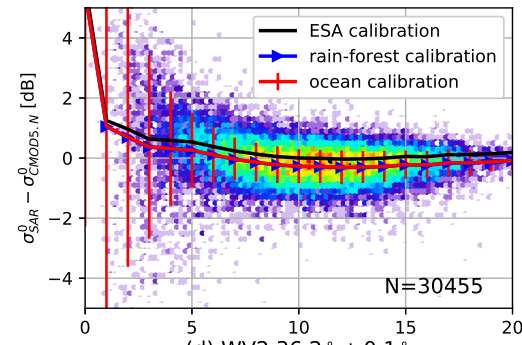

(d) WV2 $36.2^{\circ}+0.1$

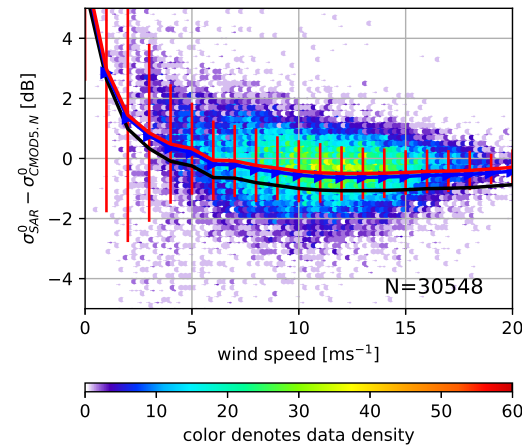

(b) WV1 $23.6^{\circ} \pm 0.1^{\circ}$

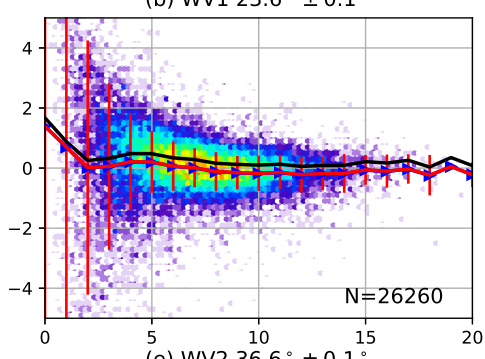

(e) WV2 $36.6^{\circ} \pm 0.1^{\circ}$

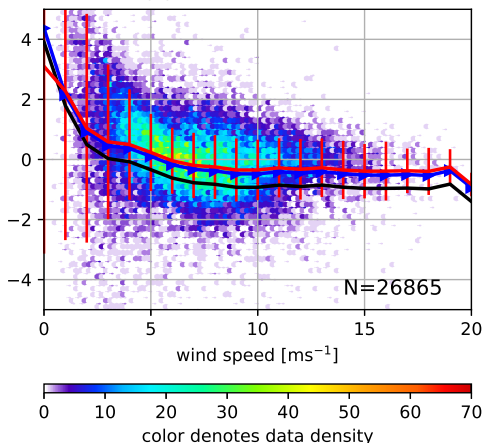

(c) WV1 $24.2^{\circ} \pm 0.1^{\circ}$

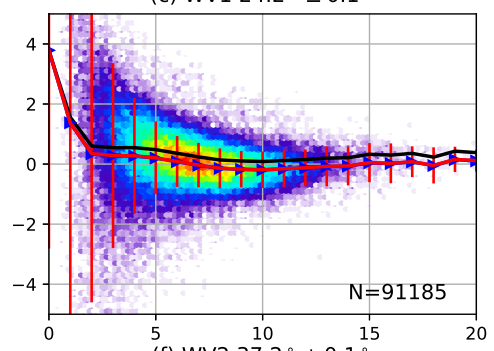

(f) WV2 $37.2^{\circ} \pm 0.1^{\circ}$

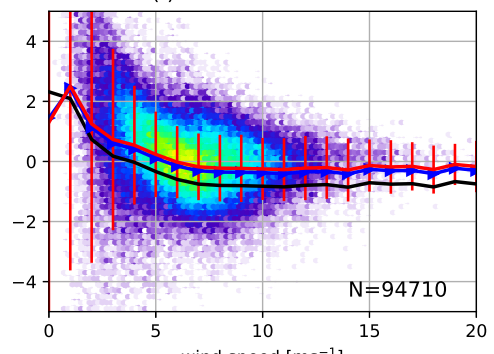

wind speed $\left[\mathrm{ms}^{-1}\right]$



Fig. 8. Re-calibrated NRCS residuals $\left(\sigma_{S A R}^{0}-\sigma_{C M O D 5 . N}^{0}\right)$ relative to wind speeds. Data (in color) are re-calibrated NRCS by ocean calibration method. Black lines are the replica of Fig. 3 and blue (red) lines are mean re-calibrated NRCS residuals using Amazon rain-forest (ocean calibration) method. The wind speed bin is $1 \mathrm{~ms}^{-1}$. The error bar represents 1 standard deviation of NRCS difference within each bin.

and ECMWF winds enables a radiometric calibration. Both analyses reveal the necessity for a re-calibration, notably for WV2. Comparable correction factors are obtained with the two methods. Applying these two methods significantly reduces the NRCS residual presented in Fig. 3.

We further discuss the limitations and possible improvements for these two methods. First a seasonal variation of rainforest backscattering is expected to affect the radiometric calibration accuracy for active sensors operating at C-band [28]. Indeed, there are two main seasons in the Amazon rain-forest: the flooded season and the dry season [30]. Precipitation and leaves on the trees and plants differs greatly from one season to another. Since incident radar pulses interact primarily with the crown area, the seasonal change of precipitation and leaves is supposed to correspondingly affect the radar return. According to previous studies based on C-band ERS-1/2 scatterometers and Radarsat-1 SAR observations, an annual variation on the order of $0.15 \mathrm{~dB}$ has been found [28]. But the geophysical source of this seasonal change remains undetermined. The collected IW products over rain-forest are used to assess the seasonal variation of $\gamma^{0}$ as observed by Sentinel-1. The $\gamma^{0}$ data points at spatial resolution of $10 \mathrm{~km}$ are weekly averaged and presented in Fig. 9 as function of time. In spite of a large standard deviation (due to too few data points), a weak variation of backscattering with respect to time is observed. This variation is more a drift compared to a clear seasonal pattern, which might be due to limited duration of S-1 data.

As discussed in section IV-C, a remaining bias is observed for $\theta=36.2^{\circ}$. As shown in Fig. 1, these incidence angles correspond to latitudes larger than $40^{\circ} \mathrm{S}$. This could be due to ECMWF wind accuracy for the performance of ocean calibration, the negative NRCS residual found in Fig. 8 (d)
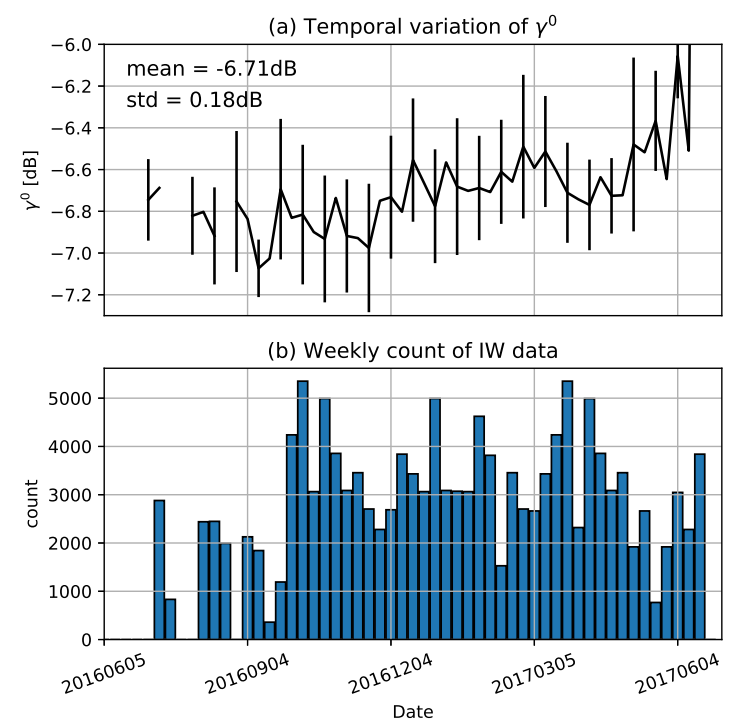

Fig. 9. (a) Weekly average of IW $\gamma^{0}$ over Amazon rain-forest over time. The error bar indicates position of mean value and standard deviation. (b) Weekly count of processed IW points. Bin size of $\gamma^{0}$ is $0.1 \mathrm{~dB}$.

is discussed in detail. We examined the ECMWF wind speed relative to buoy wind speed in the Southern Ocean. Wind speeds from the Argentine Basis buoy and Southern Ocean buoy operated by Ocean Observatories Initiatives (OOI) are used. The buoy anemometers measure winds at $5 \mathrm{~m}$ above the ocean surface and we converted the wind speed to U10 with $\log$ wind profile assuming neutral atmospheric stability. The wind speed is available every 65 seconds and are further hourly-averaged around the SAR passing time. The collocation 
in space between SAR and buoy is limited to $100 \mathrm{~km}$. Fig. 10 (a) compare collocated ECMWF winds and relative to buoy wind speeds. ECMWF wind speeds are overall consistent the buoy wind speed. The re-calibrated NRCS residuals for both ECMWF winds and buoy winds are plotted in Fig. 10 (b). For buoy winds higher than $10 \mathrm{~ms}^{-1}$, the mean NRCS residual for ECMWF winds are $-0.1128 \mathrm{~dB}$, roughly consistent with Fig. 8 (d). We also compared the wind retrieval with buoy winds and found that winds bias (SAR winds - buoy winds) using re-calibrated NRCS is improved to $0.002 \mathrm{~ms}^{-1}$ from $-0.5 \mathrm{~ms}^{-1}$ using ESA-calibrated NRCS. Yet, the limited collocation number is insufficient to unambiguously reproduce the NRCS residuals trend with wind speed. To date, in-situ data in such high latitudes are very rare and a complementary analysis will be done in the future when more collocations are available.
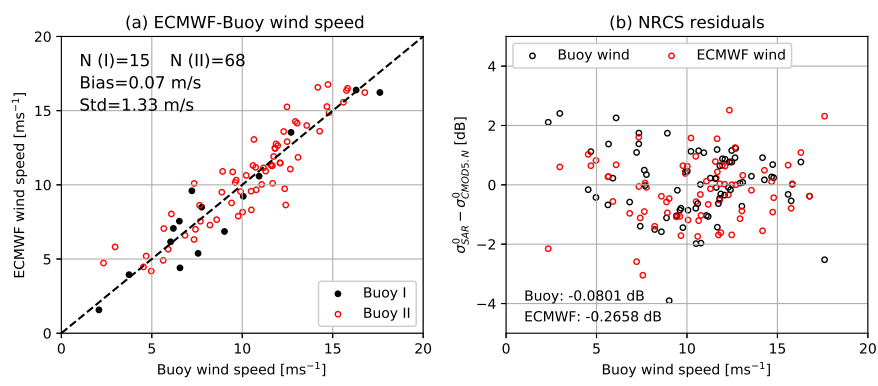

Fig. 10. (a) Comparison of ECMWF wind speed with buoy wind speed for both Argentine Basis buoy (Buoy I) and Southern Ocean buoy (Buoy II);(b) WV2 re-calibrated NRCS residuals using ocean calibration. The annotated NRCS residual is calculated to demonstrate the steady residual as shown in Fig.8(d). The buoy wind speed is an hourly average around the SAR passing time.

Overall, ocean calibration proves its efficiency through the comparable improvement while using a short duration of acquisitions. Because WV is not the routine acquisition mode over Amazon rain-forest, as a result, a small amount of images are inadequate to derive monthly correction constant. While ocean calibration uses regular acquisitions over open ocean, making a monthly calibration possible. It therefore allows to monitor the shift in backscattering signal and to accordingly compensate it over time. Furthermore, the ocean calibration per incidence angle is necessary as incidence angle is latitude dependent. These subjects are beyond scope of the present manuscript and will be addressed in the future.

\section{CONCLUSION}

Accurate radiometric calibration of a SAR is fundamental to various applications for land and ocean studies. In this manuscript, we assessed radiometric calibration accuracy of S1A WV through comparisons of SAR-measured NRCS with CMOD5.N prediction over open ocean and at global scale. WV1 and WV2 exhibit distinct calibration accuracy: WV1 is overall consistent with CMOD5.N predictions while $\mathrm{WV} 2$ shows a considerable deviation from CMOD5.N. Two recalibration methods are then carried out by examining the $\gamma^{0}$ profile over Amazon rain-forest as well as ocean calibration. These two methods give comparable correction constants for WV1 and WV2, respectively. When the corrections are applied, the NRCS residuals between re-calibrated NRCS and CMOD5.N predicts are greatly reduced towards zero. By comparison, ocean calibration is more advantageous than the rainforest calibration method, showing greater improvement of NRCS residuals as well as being more practical to implement.

Calibration strategy for S-1A WV is completely different from that for the other three imaging modes (SM, IW, and EW), which are designed to calibrate over ground transponders as well as Amazon rain-forest [29]. The assessment of WV NRCS suggests that it is necessary to reconsider the WV Level-1 SLC products calibration strategy. The ocean calibration method is efficient and practical to implement. As such, a considerate re-calibration of both S-1A and S-1B WV NRCS can be carried out per cycle.

The calibrated data should allow to improve the accuracy of wind retrieval, increase its consistency with ASCAT missions and to further study the impact of sea state on SAR-measured wind at higher incidence angles [7]. However, it should be noted that this re-calibration implies an additional correction to NRCS calibrated by ESA. To resolve this problem from the root, we therefore suggest to revisit and update the calibration procedure in the ESA Instrument Processing Facility.

\section{ACKNOWLEDGMENT}

We would like to acknowledge the ESA project: Sentinel1 A Mission Performance Center for access of Sentinel-1 Wave Mode and Interferometric Wide Swath data. Copernicus Sentinel data $(2016,2017)$ are used in this study. ECMWF forecast winds were obtained in the framework of Sentinel-1 A Mission Performance Center ESA project. ECMWF forecast winds are publicly available (ecmwf.int). The Ocean Observatories Initiatives' (OOI) buoy data are available (ooinet.oceanobservatories.org). Huimin LI would like to thank PhD financial support from China Scholarship Council (CSC).

\section{REFERENCES}

[1] D. Geudtner, R. Torres, P. Snoeij, M. Davidson, and B. Rommen, "Sentinel-1 system capabilities and applications," in 2014 IEEE Geoscience and Remote Sensing Symposium, July 2014, pp. 1457-1460.

[2] S. Lehner, J. Schulz-Stellenfleth, B. Schättler, H. Breit, and J. Horstmann, "Wind and wave measurements using complex ers-2 sar wave mode data," vol. 38, no. 5, pp. 2246-2257, 2000.

[3] K. F. Hasselmann, B. Chapron, L. Aouf, F. Ardhuin, F. Collard, G. Engen, S. Hasselmann, P. Heimbach, P. Janssen, H. Johnsen, H. Krogstad, S. Lehner, J.-G. Li, X.-M. Li, W. Rosenthal, and J. Schulz-Stellenfleth, "The ers sar wave mode: A breakthrough in global ocean wave observations," European Space Agency, Tech. Rep. 1326, 167-197, 2013.

[4] R. Torres, P. Snoeij, D. Geudtner, D. Bibby, M. Davidson, E. Attema, P. Potin, B. Rommen, N. Floury, M. Brown, I. N. Traver, P. Deghaye, B. Duesmann, B. Rosich, N. Miranda, C. Bruno, M. L'Abbate, R. Croci, A. Pietropaolo, M. Huchler, and F. Rostan, "Gmes sentinel-1 mission," Remote Sensing of Environment, vol. 120, no. Supplement C, pp. 9 - 24, 2012, the Sentinel Missions - New Opportunities for Science. [Online]. Available: http://www.sciencedirect.com/science/article/pii/S0034425712000600

[5] J. Horstmann, H. Schiller, J. Schulz-Stellenfleth, and S. Lehner, "Global wind speed retrieval from SAR," vol. 41, no. 10, pp. 2277-2286, 2004.

[6] J. Schulz-Stellenfleth and S. Lehner, "Spaceborne synthetic aperture radar observations of ocean waves traveling into sea ice," Journal of Geophysical Research, vol. 107, no. C8, 2002. 
[7] J. E. Stopa, A. A. Mouche, B. Chapron, and F. Collard, "Sea state impacts on wind speed retrievals from c-band radars," IEEE Journal of Selected Topics in Applied Earth Observations and Remote Sensing, vol. 10, no. 5, pp. 2147-2155, May 2017.

[8] H. Wang, J. Yang, A. Mouche, W. Shao, J. Zhu, L. Ren, and C. Xie, "Gf3 sar ocean wind retrieval: The first view and preliminary assessment," Remote Sensing, vol. 9, no. 7, 2017.

[9] B. Chapron, H. Johnsen, and R. Garello, "Wave and wind retrieval from sar images of the ocean," Annales Des Télécommunications, vol. 56, no. 11, pp. 682-699, Nov 2001. [Online]. Available: https://doi.org/10.1007/BF02995562

[10] M. Shimada, O. Isoguchi, T. Tadono, and K. Isono, "Palsar radiometric and geometric calibration," IEEE Transactions on Geoscience and Remote Sensing, vol. 47, no. 12, pp. 3915-3932, 2009.

[11] S. Sharma, G. Dadhich, M. Rambhia, A. K. Mathur, R. Prajapati, P. Patel, and A. Shukla, "Radiometric calibration stability assessment for the risat-1 sar sensor using a deployed point target array at the desalpar site, rann of kutch, india," International Journal of Remote Sensing, vol. 38 , no. 23 , pp. $7242-7259,2017$. [Online]. Available: https://doi.org/10.1080/01431161.2017.1371858

[12] R. Crapolicchio, G. D. Chiara, A. Elyouncha, P. Lecomte, X. Neyt, A. Paciucci, and M. Talone, "Ers-2 scatterometer: Mission performances and current reprocessing achievements," IEEE Transactions on Geoscience and Remote Sensing, vol. 50, no. 7, pp. 2427-2448, July 2012.

[13] M. Gupta, A. Sharma, and B. Kartikeyan, "Evaluation of risat-1 sar radiometric calibration using extended amazon rainforest," Journal of the Indian Society of Remote Sensing, vol. 45, no. 2, pp. 195-207, Apr 2017. [Online]. Available: https://doi.org/10.1007/s12524-016-0582-5

[14] R. Crapolicchio and P. Lecomte, "On the stability of amazon rainforest backscattering during the ers-2 scatterometer mission lifetime," in Proceedings of the SAR Workshop: CEOS Committee on Earth Observation Satellites, Toulouse, France, October 1999.

[15] PCS Team, "Ers-2 wind scatterometer cyclic report: from 28th june 1999 to 2nd august 1999 cycle 44," ESA ESRIN, Tech. Rep., 1999.

[16] J. Verspeek, M. Portabella, A. Stoffelen, and A. Verhoef, "Calibration and validation of ascat winds," Ocean and Sea Ice SAF, Tech. Rep., 2013.

[17] European Space Agency, "User guide," https://sentinel.esa.int/web/sentinel/user-guides/sentinel-1sar/acquisition-modes/wave. $\quad$ [Online]. Available: https://sentinel.esa.int/web/sentinel/user-guides/sentinel-1sar/acquisition-modes/wave

[18] R. Atlas, R. N. Hoffman, S. C. Bloom, J. C. Jusem, and J. Ardizzone, "A multiyear global surface wind velocity dataset using ssm/i wind observations," Bulletin of the American Meteorological Society, vol. 77, no. 5, pp. 869-882, 1996. [Online]. Available: https://doi.org/10.1175/1520-0477(1996)077;0869:AMGSWV ¿2.0.CO;2

[19] A. Mouche and B. Chapron, "Global c-band envisat, radarsat2 and sentinel-1 sar measurements in copolarization and cross-polarization," Journal of Geophysical Research: Oceans, vol. 120, no. 11, pp. 7195-7207, 2015. [Online]. Available: http://dx.doi.org/10.1002/2015JC011149

[20] European Space Agency, "Level-1 radiometric calibration," [Online; accessed 11-December-2017]. [Online]. Available: https://sentinels.copernicus.eu/web/sentinel/radiometriccalibration-of-level-1-products

[21] Collecte Localisation Satellites (CLS), "Sentinel-1a and -1b annual performance report 2016," CLS, Tech. Rep., 2017.

[22] H. Hersbach, "Cmod5.n: A c-band geophysical model function for equivalent neutral wind." April 2008.

[23] G. K. Carvajal, L. E. B. Eriksson, and L. M. H. Ulander, "Retrieval and quality assessment of wind velocity vectors on the ocean with c-band sar," IEEE Transactions on Geoscience and Remote Sensing, vol. 52, no. 5, pp. 2519-2537, May 2014.

[24] Jagdish, S. V. V. A. Kumar, A. Chakraborty, and R. Kumar, "Validation of wind speed retrieval from risat-1 sar images of the north indian ocean," Remote Sensing Letters, vol. 9, no. 5, pp. 421-428, 2018. [Online]. Available: https://doi.org/10.1080/2150704X.2018.1430392

[25] Ocean and Sea Ice SAF, "Algorithm theoretical basis document for the osi saf wind products," June 2016.

[26] J. E. Stopa and A. Mouche, "Significant wave heights from sentinel-1 sar: Validation and applications," Journal of Geophysical Research: Oceans, vol. 122, no. 3, pp. 1827-1848, 2017. [Online]. Available: http://dx.doi.org/10.1002/2016JC012364

[27] D. G. Long and G. B. Skouson, "Calibration of spaceborne scatterometers using tropical rain forests," IEEE Transactions on Geoscience and Remote Sensing, vol. 34, no. 2, pp. 413-424, Mar 1996.
[28] R. Hawkins, E. Attema, R. Crapolicchio, P. Lecomte, J. Closa, P. Meadows, and S. Srivastava, "Stability of amazon backscatter at c-band: Spaceborne results from ers-1/2 and radarsat-1," in Proceedings of the SAR Workshop: CEOS Committee on Earth Observation Satellites, Toulouse, France, October 1999.

[29] M. Schwerdt, K. Schmidt, N. T. Ramon, G. C. Alfonzo, B. J. Dring, M. Zink, and P. Prats-Iraola, "Independent verification of the sentinel-1a system calibration," IEEE Journal of Selected Topics in Applied Earth Observations and Remote Sensing, vol. 9, no. 3, pp. 994-1007, March 2016.

[30] P. M. Brando, S. J. Goetz, A. Baccini, D. C. Nepstad, P. S. A. Beck, and M. C. Christman, "Seasonal and interannual variability of climate and vegetation indices across the amazon," Proceedings of the National Academy of Sciences, vol. 107, no. 33, pp. 14685-14690, 2010. [Online]. Available: http://www.pnas.org/content/107/33/14685.abstract



Huimin Li received a B.S. and M.S. in Ocean Information Detection from Ocean University of China, Qingdao, China in 2013 and 2015, respectively. She is currently a $\mathrm{PhD}$ student in the Laboratoire $\mathrm{dO}$ canographie Physique Spatiale, Institut Francais de Recherche pour IExploitation de la Mer, Plouzane, France.

Her thesis mainly focuses on a better understanding of SAR imaging mechanism over ocean waves. She also works on analysis of SAR images to obtain simultaneous observations of wind, wave

and current.

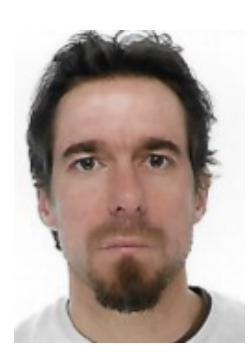

Alexis Mouche received the Masters degree in physics for remote sensing from the University of Pierre et Marie Curie, Paris, France, in 2002, and the Ph.D. degree in physics with a focus on ocean remote sensing from ETP/IPSL/CNRS (National Research Center), Paris, France, in 2005.

In 2006, he joined the Laboratoire dOcanographie Spatiale, IFREMER for 2 years with a Postdoctoral Position granted by the French spatial agency (CNES) to work on the ocean dynamics as observed at high resolution. From 2008 to 2013, he worked as a Research Engineer with BOOST-Technologies and CLS (CNES and IFREMER subsidiary) to develop algorithms for wind, waves, and current measurements with synthetic aperture radar from space. Since January 2014, he has been with the Laboratoire dOcanographie Physique and Spatiale, Ifremer. He is currently a Research Scientist at IFREMER, Brest, France. His principal research interests include the interactions of electromagnetic and oceanic waves for ocean remote sensing applications.



Justin Stopa received a M.S. and Ph.D degrees in ocean engineering from the University of Hawaii at Manoa, Honolulu, Hawaii, USA in 2007 and 2013 respectively. His dissertation focused on the wind and wave climate. From 2014-2018 he was a research oceanographer at the Laboratory of Ocean Physics and remote Sensing (LOPS) in Plouzane, France.

Now he is currently an assistant professor at the University of Hawaii at Manoa in the department of Ocean Resources and Engineering. He has expertise in physical oceanography, operational sea state prediction, oceanic remote sensing, spectral wave modeling, and applied statistics. His principle research interests include ocean waves, remote sensing, and climate (www.swelltracker.org). 


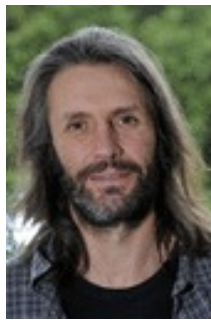

Bertrand Chapron received the Ph.D. degree in fluid mechanics from AixMarseille University, Marseille, France, in 1988.

$\mathrm{He}$ is currently a Senior Research Scientist with the Laboratoire dOcanographie Physique Spatiale, Institut Francais de Recherche pour lExploitation de la Mer, Plouzane, France. He has been the Co-Investigator or Principal Investigator in several ESA (ENVISAT RA2, ASAR, and SMOS), NASA, and CNES (TOPEX and JASON) projects. He contributed to the development of several algorithms for geophysical parameter retrieval from altimeters, radiometers, or SAR. He is co-responsible for the ENVISAT ASAR-Wave Mode algorithms and scientific preparation for the ENVISAT and S1 wind, wave, and current. Recently, he collaborated in studies that demonstrated the high potential of radiometers for wind retrieval in extreme conditions and the possibility of doing directional ocean waves spectrum from Sentinel-2. He has authored over 80 publications in refereed journals in applied mathematics, physical oceanography (upper ocean dynamics), and electromagnetic wave theory and its application to ocean surface remote sensing. 